\title{
5 Research Square

\section{Human Amniotic Mesenchymal Stem Cells Inhibit aGVHD in Humanized Mice by Regulating the Balance of Treg and T Effector Cells}

\section{Ya Gao}

Southern Medical University Nanfang Hospital

\section{Wei-Ru Li}

Southern Medical University Nanfang Hospital

\section{Xiao-Yin Bu}

Southern Medical University Nanfang Hospital

\section{Ying Xu}

Southern Medical University Nanfang Hospital

\section{Sheng-Chun Cai}

Southern Medical University Nanfang Hospital

\section{Jin-Man Zhong}

Southern Medical University Nanfang Hospital

\section{Mei-Xue Du}

Southern Medical University Nanfang Hospital

\section{Hai-Tao Sun}

nan fang yi ke da xue zhu jiang yi yuan: Zhujiang Hospital

\section{Li-Ping Huang}

Southern Medical University Nanfang Hospital

\section{Yong-Jian He}

Southern Medical University Nanfang Hospital

\section{Xiu-Mei Hu}

Southern Medical University Nanfang Hospital

\section{Qi-Fa Liu}

Southern Medical University Nanfang Hospital

\section{Hua Jin}

Southern Medical University Nanfang Hospital

\section{Qian Wang}

nan fang yi ke da xue zhu jiang yi yuan: Zhujiang Hospital

\section{Bao-hong Ping ( $\nabla$ nfyypingbaohong66@163.com )}

Southern Medical University Nanfang Hospital https://orcid.org/0000-0003-1177-5904 
Research

Keywords: Acute graft versus host disease, humanized mouse model, amniotic mesenchymal stem cells, balance of Treg vs. T effector cells

Posted Date: June 8th, 2021

DOI: https://doi.org/10.21203/rs.3.rs-581864/v1

License: (c) (i) This work is licensed under a Creative Commons Attribution 4.0 International License.

Read Full License 


\section{Abstract}

Background: Acute graft versus host disease (aGVHD) remains a leading cause of transplant-related mortality following allogeneic haematopoietic cell transplantation(allo-HCT). Although previous studies indicated that mesenchymal stem cells (MSCs) may be a salvage therapeutic agent for aGVHD, the mechanism is not yet fully clear. Human amniotic mesenchymal stem cells (hAMSCs) is a novel MSCs, compared with bone marrow mesenchymal stem cells, it has the advantage of being non-invasive, and also has stronger proliferation ability than that of BM-MSCs and equivalent immune regulation ability as BM-MSCs. The aim of this study was to explore the therapeutic efficacy and underlying mechanisms of human amniotic mesenchymal stem cells transplantation for the humanized aGVHD mouse model.

Methods: We established a humanized aGVHD mouse model by transplanting human peripheral blood mononuclear cells (PBMCs) into NOD-Prkdc ${ }^{\text {scid IL2ry }}$ null (NPG) mice, hAMSCs collected from discarded placenta of healthy pregnant women after delivery. Mice were divided into control group (untreated), aGVHD group, and hAMSCs treatment group, the hAMSCs labeled with GFP were administered to aGVHD mice to explore the homing ability of hAMSCs. T effector and Treg cell levels and cytokines of each group in target organs were detected by flow cytometry and cytometric bead array (CBA) respectively.

Results: We successfully established a humanized aGVHD mouse model using NPG mice. The hAMSCs have the ability to inhibit aGVHD in this mouse model through reduced villous blunting and lymphocyte infiltration into the lamina propria of the gut while reducing vascular endothelialitis and lymphocyte infiltration into the parenchyma of the liver and lung. hAMSCs suppressed xenogenesis CD3+CD4+ T and CD3+CD8+ $T$ cell expression and increased the proportion of Treg cells, and besides, hAMSCs can reduce the levels of IL-17A, INF- $y$, TNF, and IL-2 in aGVHD target organs.

Conclusions: The NPG murine environment was capable of activating human T cells to produce aGVHD pathology to mimic aGVHD as in humans. The hAMSCs controlled aGVHD by decreasing inflammatory cytokine secretion within target organs by modulating the balance of Treg and T effector cells in humanized mice.

\section{Introduction}

Graft-versus-host disease (GVHD) is reported as a syndrome in which donor immunocompetent cells recognize and attack host tissues in immunocompromised allogeneic recipients after hematopoietic stem cell transplantation (HSCT) [1, 2]. Steroids are the appropriate first line treatment for this disease; however, there are many treatment-related complications [3]. Besides, around half of patients develop aGVHD, even when preventive measures are adequately used [4]. Thus, there is an urgent need to develop more potent immunosuppressive treatment strategies for patients suffering from acute steroid refractory aGVHD while maintaining the graft versus tumor effect to avoid a potential rise in relapse-related mortality. 
Mesenchymal stem cells (MSCs) are non-hematopoietic adult multipotent progenitor cells that can be isolated from various adult tissues. Human amniotic mesenchymal stem cells (hAMSCs) are a type of MSCs that can be easily and safely isolated from the amniotic membrane of healthy pregnant women (medical waste) and have been recognized as one of the most promising stem cells in the field of regenerative medicine [5]. The hAMSCs express CD90, CD105, and CD73 but not hematopoietic cell markers such as CD45, CD34, HLA-DR, and CD11b. The hAMSCs possess immunomodulatory properties that are thought to enable damaged tissues to form a balanced inflammatory and regenerative microenvironment in the presence of vigorous inflammation [6]. Furthermore, the immunomodulatory activity of hAMSCs is equal to or higher than that of human bone marrow MSCs [7]. Thus, the active proliferative potential, low immunogenic profile, and anti-inflammatory function of hAMSCs can be beneficial in the treatment of inflammation-related diseases and immunosuppression. Yamahara et al. found that steroidrefractory aGVHD infusion of hAMSCs was safe and effective [8]. Our previous study demonstrated that hAMSCs induced Th1 cells into Th2 cells in vitro [9]. However, there are few reports about the mechanisms by which hAMSCs exert their therapeutic effects in vivo.

Due to ethical constraints, relevant research cannot be conducted on humans and GVHD pathogenesis has been mostly studied in mouse models of transplantation. Until recently, C57/BI6 (H2b) donors in $\mathrm{BALB} / \mathrm{c}(\mathrm{H} 2 \mathrm{~d})$ recipients have been widely used in the establishment of aGVHD model mice. However, mouse aGVHD models have some limitations. To generate a system whereby human T cell-mediated aGVHD can be studied and manipulated in vivo, xenogeneic transplant models have been rapidly developed [10]. Currently, the most popular immune deficient mouse strains, NOD/SCID-IL2Ry ${ }^{-1-}$, NOD.Cg-

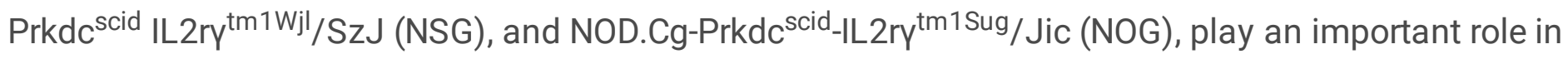
the study of the immunological pathological mechanisms of GVHD and the improvement of therapy. Those mice lack T, B, and NK cells, and also have reduced macrophage and dendritic cell function that allow efficient human peripheral blood mononuclear cells (PBMCs) engraftment. Transplantation of PBMCs can cause aGVHD syndrome because human APCs process mouse antigens and present them in the presence of class II MHC [11]. Compared to mouse models of aGVHD, the humanized model has many advantages such as the use of human cells to induce and control xenogeneic (xeno)-aGVHD, the possibility of using donors with high genetic diversity, and the possibility of using older donors previously exposed to various pathogens [12]. Here, we used NOD-Prkdc ${ }^{\text {scid IL2ry }}{ }^{\text {null }}$ (NPG) mice to establish a xenogeneic aGVHD humanized model and investigated the therapeutic potential of hAMSCs in preventing aGVHD in vivo. We demonstrated that hAMSCs ameliorated aGVHD via the addition of Treg cells and reduction of inflammatory cytokine secretion in target organs. Our results supported further application of human amniotic mesenchymal stem cells to prevent and treat aGVHD clinically.

\section{Methods}

\section{Isolation and culture of cells}


Human placentas were obtained from caesarean sections from healthy women with informed consent. The amnion layer was separated from the chorion layer and washed several times with phosphatebuffered saline (PBS), cut into $1.0 \mathrm{~cm}^{2}$ pieces, and digested with $0.25 \%$ trypsin at $37^{\circ} \mathrm{C}$ for $30 \mathrm{~min}$. Amniotic epithelial cells were removed gently and the amnion was digested again under the same conditions. Finally, the amnion was washed with PBS and cut into $1 \mathrm{~mm}^{2}$ pieces and cultured in a-MEM (1x; Gibco, USA, C12571) supplemented with 10\% fetal bovine serum (FBS; Gibco, Brazil, A31608-02), $0.01 \mathrm{mM} / \mathrm{mL}$ sodium pyruvate (Corning, USA, 15719005), $100 \mathrm{U} / \mathrm{mL}$ penicillin streptomycin (Corning, USA, 15719005), and $0.01 \mathrm{mM} / \mathrm{mL}$ nonessential amino acids (Solarbio, China, N1250) at $37^{\circ} \mathrm{C}$ and $5 \%$ $\mathrm{CO}_{2}$. Cells were digested and passaged when the cell density reached $80-90 \%$ after 5 days. Cells were cultured in culture flasks $(3 \mathrm{~mL})$ at a density of $1 \times 10^{6} / \mathrm{mL}$. hAMSCs used in experiments were third- to fifth-passage. A suspension of P3 hAMSCs was counted and adjusted to $1 \times 10^{6}$ cells $/ \mathrm{mL}$.

\section{Identification of human amniotic mesenchymal stem cells}

For identification, cells were incubated with mouse anti-human CD90-PE antibody (BD Pharmingen, catalog number: 561970), mouse anti-human CD105-PE antibody (eBioscience, catalog number: 12-105742), mouse anti-human CD73-PE antibody (BD Pharmingen, catalog number: 561014), mouse anti-human CD45-PE antibody (BD Pharmingen, catalog number: 560975), mouse anti-human CD11b-PE-Cy ${ }^{\text {TM }} 7$ antibody (BD Pharmingen, catalog number: 561098), mouse anti-human CD34-PE antibody (BD Pharmingen, catalog number: 560941), and mouse anti-human HLA-DR-PE antibody (BD Pharmingen, catalog number: 560943 ) at $4^{\circ} \mathrm{C}$ for $30 \mathrm{~min}$. Cells were washed with PBS, adjusted to $300 \mu \mathrm{L}$, and analyzed by flow cytometry.

\section{Adipogenic and osteogenic differentiation}

To analyze adipogenic and osteogenic differentiation, passage three hAMSCs were seeded at a density of $1.5 \times 10^{5}$ cells/well in a six-well plate. When the cells reached $85-90 \%$ confluence, cells were incubated in human MSCs adipogenic differentiation medium (Pythonbio, catalog number: 20191008), for 21 days; the adipogenic induction medium was replaced every 3 days. Oil red O (Solarbio, catalog number: G1260) staining was performed to assess the differentiation potential. For osteogenic differentiation, hAMSCs were cultured with human MSCs osteogenic differentiation medium (Pythonbio, catalog number: 20191009) for 21 days; the induction medium was replaced every 3 days. The differentiation potential for osteogenesis was assessed by Alizarin Red (pH 4.2, 40 mM) (Solarbio, catalog number: G1452) staining.

\section{Animals}

NPG mice 8-10 weeks of age were obtained from Beijing Vitalstar Biotechnology Co., Ltd. (laboratory animal production license no. SCXK2019-0002). Animals were housed in a specific pathogen-free facility in microisolator cages, given autoclaved food, and maintained on acidified autoclaved water and a solution of gentamicin. All animal experiments were approved by the laboratory animal ethics review committee of Southern Medical University. All animal procedures were in accordance with the National Institute regarding laboratory animal care and use. 


\section{Human PBMCs collection}

Human PBMCs were collected from healthy volunteers. Each donor provided written informed consent. Peripheral blood was collected in sodium citrate and PBMCs were isolated from peripheral blood by Ficoll-Hypaque (Solarbio) density centrifugation, washed in PBS, suspended in red blood cell lysis buffer (Solarbio) at $4^{\circ} \mathrm{C}$ for $15 \mathrm{~min}$, washed again in PBS, and suspended in PBS for tail vein injection into NPG mice.

\section{Induction of xenogeneic aGVHD in NPG mice}

Mice were given $200 \mathrm{cGy}$ irradiation 3-4h before cell injection followed by tail vein injection of $3 \times 10^{6}$ PBMCs suspended in 500 $\mu$ L PBS unless indicated otherwise. During all experiments, each mouse was graded according the xeno-aGVHD clinical scoring system (Table 1). The symptoms of xeno-GVHD included weight loss, hunched posture, ruffled fur, reduced mobility, and diarrhea. Mice survived to the 28 day endpoint and those that suffered from severe xeno-GVHD (weight loss of $25 \%$, severe hunched posture, severe ruffled fur, less or no mobility, or hematochezia) were euthanized.

\section{GFP-labeled hAMSCs and hAMSCs tracing in vivo}

The hAMSCs were added to a 24-well plate at a concentration of $3 \times 10^{5} / \mathrm{mL}, 500 \mu \mathrm{L}$ per well. The 24 -well plate was incubated at $37^{\circ} \mathrm{C}$ and $5 \% \mathrm{CO}_{2}$. After $24 \mathrm{~h}$, the culture media was disposed, and $250 \mu \mathrm{L}$ of culture media and $1.6 \mu \mathrm{L}$ of GFP-pseudovirion (Hanbio) were added; the rest of the $250 \mu \mathrm{L}$ culture media was added $4 \mathrm{~h}$ later and the plate was incubated. After $24 \mathrm{~h}$, the culture media was disposed, $500 \mu \mathrm{L}$ of culture media was added and the plate was incubated. GFP-labeled hAMSCs were identified by fluorescence microscope after 2-3 days. The suspension of GFP-labeled hAMSCs was adjusted to $5 \times$ $10^{5}$ cells suspended in $500 \mu \mathrm{L}$ PBS and injected into NPG mice via the tail vein. These mice were euthanized after $24 \mathrm{~h}$ and $72 \mathrm{~h}$. Blood and tissues such as the liver, spleen, lung, and gut were collected. For immunofluorescence, target organs were fixed with $4 \%$ paraformaldehyde for $24 \mathrm{~h}$ and dehydrated with $30 \%$ sucrose. After more than $48 \mathrm{~h}$, target organs were embedded with a frozen slicer at $-25^{\circ} \mathrm{C}$ to generate slices. Recipient cells were distinguished by DAPI counterstaining.

\section{Histopathological analyses}

Tissues were collected at the time of necropsy, fixed in $10 \%$ buffered formalin, and embedded in paraffin. For routine histology, sections were stained with hematoxylin and eosin. For immunohistochemistry, sections were heated at $60^{\circ} \mathrm{C}$ for $20 \mathrm{~min}$, deparaffinized, and hydrated with xylene and alcohol baths for staining. The slides were heated in $0.01 \mathrm{M}$ citrate-buffer solution $(\mathrm{pH} \mathrm{6.0)}$ for $10 \mathrm{~min}$ in a microwave oven, then cooled down to room temperature. Immunohistochemical staining was performed with a monoclonal antibody specific for human CD45 (Abcam, USA). For immunofluorescence, target organs were fixed with $4 \%$ paraformaldehyde for $24 \mathrm{~h}$ and dehydrated with $30 \%$ sucrose. After more than $48 \mathrm{~h}$, target organs were embedded in a frozen slicer at $-25^{\circ} \mathrm{C}$ to generate slices. Recipient cells were distinguished by DAPI counterstaining. 


\section{Flow cytometric analysis}

Mouse anti-human CD3-PE-Cyanine7 antibody (eBioscience, catalog number: 25-0038-42), mouse antihuman CD4-FITC antibody (BD Pharmingen, catalog number: 340133), mouse anti-human CD8-PE (BD Pharmingen, catalog number: 340046), mouse anti-human CD25-APC antibody (Biolegend, catalog number: 302610), and mouse anti-human Foxp3-PE (eBioscience, catalog number: 12-4777-42) were used. Peripheral blood, livers, spleens, lungs and guts were collected at the time of necropsy and analyzed by flow cytometry. Single cell suspensions were obtained by grinding the liver, spleen, lung, and gut, and blood samples were processed with red blood cell lysis buffer (Solarbio) at $4^{\circ} \mathrm{C}$ according to the protocol. All samples were stained with antibodies or isotype matched control IgG for $30 \mathrm{~min}$ at $4^{\circ} \mathrm{C}$ in the dark and analyzed with a BD FACS Cantoll flow cytometer with FlowJo 10.0.

\section{Analysis of the cytokines by cytometric bead array (CBA)}

The CBA human Th1/Th2/Th17 cytokine kit (BD, catalog number: 560484) was used. Peripheral blood and single cell suspensions of each target organ were centrifuged at $4^{\circ} \mathrm{C}, 3,000 \mathrm{rpm} / \mathrm{min}$, and $20 \mathrm{~min}$. Human Th1/Th2/Th17 cytokine capture beads were mixed and added to all assay tubes. Then, $50 \mu \mathrm{L}$ of each sample was added to appropriately labeled sample tubes and $50 \mu \mathrm{L}$ of the human Th1/Th2/Th17 $\mathrm{PE}$ detection reagent was added. The assay tubes were incubated for $3 \mathrm{~h}$ at room temperature in the dark. Then, $1 \mathrm{~mL}$ of wash buffer was added to each assay tube followed by centrifugation at $200 \times g$ for 5 min. The supernatant from each assay tube was carefully aspirated and discarded, and $300 \mu \mathrm{L}$ of wash buffer was added to resuspend the bead pellet. The samples were analyzed with flow cytometry (BD FACS Cantoll) human Th1/Th2/Th17 cytokine data using FCAP Array software.

\section{Statistical analysis}

Comparisons between two means were performed using the independent samples $t$-test. Comparisons of three or more means were performed using one-way ANOVA. Survival curves were generated with the Kaplan-Meier method. $\mathrm{P}<0.05$ was considered significant. All statistical analyses were performed with GraphPad Prism 8.0.

\section{Results}

A human xenogeneic acute GVHD model was successfully established in NPG mice.

To mimic GVHD in humans, we established xeno-aGVHD humanized mice using NPG mice. Two groups of NPG mice were generated: PBS only (control group) ( $n=3)$ or PBMCs $\left(3 \times 10^{6}\right.$ cells each mouse) following $200 \mathrm{cGy}$ irradiation (aGVHD group) $(n=6)$ [Fig. 1A]. aGVHD group developed symptoms of xeno-aGVHD, which can mimic human aGVHD, including weight loss, hunched posture, ruffed fur, reduced mobility, damaged skin, diarrhea, and hematochezia compared with control group. NPG mice that received 200 cGy irradiation prior to injection of $3 \times 10^{6}$ human PBMCs via the tail vein died 7-14 days after cell transfer..[Fig. 1B, C]. Similar to humans, NPG mice with aGVHD showed severe inflammation, 
leukocyte infiltration, necrosis, and tissue damage in target organs such as the lung, liver, and spleen [Fig. 1D]. Lump-like PBMCs infiltration was observed in the liver, spleen, and lung [Fig. 1E]. These results indicated that the aGVHD induced by xenogeneic donor hPBMCs in NPG mice was mediated mainly by human T cells. PBMCs in the blood were detected by flow cytometry, which confirmed that PBMCs were successfully injected into the body [Fig. 1F].

\section{Identification of hAMSCs in vitro and GFP labeled hAMSCs tracer cells in vivo}

Passage three hAMSCs appeared to be spindle- or polygon-shaped in morphology and reached $85-90 \%$ fusion after $48 \mathrm{~h}$ in culture [Fig. 2A]. The hAMSCs possessed osteogenic and adipogenic differentiation potentials in vitro [Fig. 2B, C]. Flow cytometry analysis of the hAMSCs surface antigen phenotype revealed that CD90, CD105, and CD73 were expressed, while CD45, CD34, HLA-DR and CD11b were not [Fig. 2D]. As the GFP gene was present in the GFP-pseudovirion, the hAMSCs transduced with pseudovirion produced a high level of GFP; the efficiency of labeling was $>80 \%(84.33 \pm 1.73)(n=6)$ [Fig. 2E]. GFP-hAMSCs were injected into NPG mice, after 24 and $72 \mathrm{~h}$, mice were euthanized separately. GFP-labeled hAMSCs were detected in the liver, spleen, lung, and gut after $24 \mathrm{~h}$. However, few GFP-labeled cells were observed in target organs except the lung after $72 \mathrm{~h}$ [Fig. 3]. The results demonstrated that after intravenous injection, hAMSCs migrated to damaged target organs.

\section{The hAMSCs alleviated clinical symptoms and improved overall survival in the murine model of aGVHD.}

To verify the immunosuppressive ability of hAMSCs in vivo, we tested hAMSCs in the xeno-aGVHD model [Fig. 4A]. As shown in Fig. 4B, hAMSCs significantly ameliorated the severity of acute GVHD in terms of weight loss and disease score. Moreover, xeno-aGVHD mice injected with hAMSCs survived for 26-28 days $(n=5)$, while the xeno-aGVHD mice that received PBS died 7-14 days after transplantation $(n=27)$. The hAMSCs significantly ameliorated the severity of acute GVHD in terms of weight loss and disease score within 28 days of transplantation $(n=27)$. Moreover, hAMSCs prevented leukocyte infiltration and reduced pathology in the lung, liver, gut, and spleen on day 6 after transplantation [Fig. 4C]. These results demonstrated that hAMSCs control aGVHD clinical symptoms in the xeno-aGVHD mouse.

\section{The hAMSCs modulate the balance of Treg versus T effector cells of target organs in humanized mice.}

To investigate the effect of hAMSCs on T effector and Treg cells, single cell suspensions of target organs were analyzed by flow cytometry. The cell concentration was $1 \times 10^{6} \mathrm{cells} / \mathrm{mL}$. We found that the proportion of CD3 $+C D 4+T$ cells in the liver, spleen, lung, and gut in hAMSCs treatment group was significantly lower than that in aGVHD group $(n=4)$ [Fig. 5A]. Consistent with the proportion of CD3 + $\mathrm{CD} 4+\mathrm{T}$ cells, the proportion of CD3 + CD8 + T cells in target organs such as liver, spleen and gut of mice in hAMSCs treatment group was also lower compared with aGVHD group $(n=3)$ [Fig. 5B]. We further found that CD $4+C D 25+$ Foxp3 + Tregs from liver, spleen, gut in hAMSCs treatment group were increased, especially in the gut $(n=3)$ [Fig. $5 \mathrm{C}$ ]. Our data supported the finding that hAMSCs treatment significantly inhibited the proportion of donor CD3 + CD $4+T$ and $C D 3+C D 8+T$ cells and increased the proportion of Treg cells in target organs from day 3 to day 6 after transplantation. 


\section{The hAMSCs control aGVHD by decreasing inflammatory cytokines secretion in target organs.}

The hAMSCs treatment significantly inhibited the secretion of human inflammatory cytokines, including IL-17A, IFN- $y$, TNF, and IL-2 from the target organs. Level of IL-2 was significantly decreased in gut and blood in hAMSCs treatment group than that in aGVHD group $(n=3)$ [Fig. 6A]. Level of IFN- $\gamma$ was significantly decreased in liver, gut and blood in hAMSCs treatment group than that in aGVHD group $(n=$ 3) [Fig. 6B]. Level of IL-17A was significantly decreased in liver, lung and gut in hAMSCs treatment group than that in aGVHD group $(n=3)$ [Fig. $6 \mathrm{C}$ ]. Level of TNF was significantly decreased in liver and lung in hAMSCs treatment group than that in aGVHD group $(n=3)$ [Fig. 6D]. Especially in the gut, an important aGVHD target organ, we found that IL-2, IFN- $\gamma$, and IL-17A were all significantly suppressed by hAMSCs. These results demonstrated that hAMSCs ameliorated human allogeneic acute GVHD by reduction of inflammatory cytokine secretion in target organs.

\section{Discussion}

Numerous reports have shown that MSCs yielded a favorable therapeutic benefit for GVHD, MSCs have been widely used in several clinical trials $[5,13]$. Nevertheless, controversy about their safety and efficacy remains. Although bone marrow (BM) is the main source of MSCs, bone marrow MSCs (BM-MSCs) are not always used because of the invasive harvesting procedure; the number of BM-MSCs declines with increasing age $[14,15]$. Placenta tissue-derived MSCs are readily available and easy to collect from a waste product, which has been reported to contain a population of multipotent stem cells exhibiting characteristics of MSCs [16]. Therefore, MSCs from amniotic membranes might be a better option than BM-MSCs. In the present study, we established a human allogeneic acute GVHD model in humanized mice by adoptive transfer of allogeneic hPBMCs into immunodeficient NOD-Prkdc ${ }^{\text {scid } / L 2 \text { ry }{ }^{\text {null }}(N P G) \text { mice }}$ $[17,18]$. The humanized aGVHD model is mediated mainly by donor T cells and characterized by disease appearance (hunching, activity, ruffling, and diarrhea), recruitment of alloreactive cells in target organs, and dysregulation of proinflammatory cytokines [17]. Although important differences remain between GVHD in humanized NPG mouse models and humans, key mechanisms of GVHD pathogenesis are shared in human and xenogeneic aGVHD. The pathogenesis of xeno-aGVHD shares important features with human GVHD such as TCR/co-stimulatory-mediated expansion of selected T cell clones that acquire mainly a Th1/Tc1 profile [19]. Furthermore, significantly increased human cell reconstitution and better immune responses, including immunoglobulin class switching and elevated human IgG responses, have been observed in NPG mice [20]. Thus, the human allogeneic acute GVHD model established here may provide a more relevant approach for studies of human immunopathogenesis and therapeutics for aGVHD after BMT. Our data showed that the murine inflammatory environment was capable of activating human T cells to produce acute GVHD pathology regardless of whether human APCs are co-transplanted. We elected to infuse $3 \times 10^{6}$ PBMCs from donors following $200 \mathrm{cGy}$ irradiation, because we previously found that infusion of $3 \times 10^{6}$ PBMCs from donors induced a moderate GVHD in that model while administration of 7-9 $\times 10^{6}$ PBMCs resulted in severe GVHD (unpublished data). In addition, the irradiation dose is proportional to the degree of tissue damage and the subsequent cytokine storm, and 
thus is directly proportional to aGVHD-related mortality in the mouse [21, 22]. Our results showed that low dose irradiation before PBMCs translation was necessary for mouse antigen exposure and to successfully induce a xeno-aGVHD model.

The hAMSCs are a novel source of stem cells that can be obtained in large quantities. Different from BMMSCs, MSCs isolated from placenta exhibit greater proliferative and differentiation potential than BMMSCs, most likely because of the early embryologic origin of amniotic membrane and placenta MSCs compared with BMMSCs $[23,24]$. The hAMSCs have intermediate levels of HLA MHC class I molecules, but do not have HLA class II antigens, FAS ligand, and the co-stimulatory molecules, and therefore, do not activate alloreactive T cells $[25,26,27]$. The hAMSCs exhibited a fibroblast-shaped morphology and adherence to plastic, our data show that the cell-surface markers of the hAMSCs were positive for CD90, CD73 and CD105 while negative for CD45, CD11b, HLA-DR and CD34. These findings met the criteria for MSCs identification by The Association of International Cell Therapy. Our observations further demonstrated GFP-labeled hAMSCs had better growth for tracers in the mouse, with a labeling rate of $84 \%$. The hAMSCs labeled with GFP fluorescence could be reproducibly and noninvasively detected by immunofluorescence in the lung, liver, spleen, and gut at day one and day three, respectively, after cell infusion via the mouse tail vein. This phenomenon may be caused by secreted cytokines such as fibroblast growth factor, chemokine receptors, and stem cell homing factors $[28,29,30]$. secreted by hAMSCs and rich marginal blood flow in that region, which are beneficial for cell migration. We also found that from day one to day three, GFP labeled cells increased gradually and were observed in the lung but also can track to the liver, spleen, and gut. However, the number of hAMSCs gradually decreased over time in those organs. It has been reported that BM-MSCs were largely trapped in the lungs, liver, and spleen with abundant capillaries after intravenous transplantation [31]. Our data were consistent with this research; we also found that hAMSCs reached target organs such as the small intestine and liver early. Therefore, hAMSCs exhibited homing ability to target damaged tissues, relieving the severity of inflammation and accelerating tissue repair. Homing of MSCs may also be associated with local microvascular changes, increased capillary permeability, hemostasis, and passive retention.

We then analyzed the function of hAMSCs in xeno-aGVHD using the humanized mice. We revealed that hAMSCs therapy reduced villous blunting and lymphocyte infiltration into the lamina propria of the gut while reducing vascular endothelialitis and lymphocyte infiltration into the parenchyma of the liver and lung. In addition, hAMSCs suppressed xenogenesis of the CD3 $+C D 4+T$ and $C D 3+C D 8+T$ cell proportions and increased the expression of Treg cells in target organs. The induction of immune tolerance involves a precise balance between activation and inhibition of $T$ cell responses, which is important in the development of aGVHD [32]. Tobin et al [33] found that BM-MSCs blocked TNF-a secretion by dendritic cells via promotion of IL-10 and IL-4 secretion, which impeded T cell differentiation into Th1 cells, directing differentiation of these cells into Treg and Th2 cells, respectively [34]. A recent study showed that AMSCs reduced the activity of human CD8 + T cells and TNF- $\alpha$ in the peripheral blood of xeno-GVHD mice. They also compared the immunomodulatory effects of AMSCs and BM-MSCs in vitro, and found that both AMSCs and BM-MSCs reduced the concentration of TNF-a and IFN- $\gamma$ expressed 
by PBMCs [35]. However, this study did not detect T cell and inflammatory cytokine levels in the target organs of aGVHD mice.

We examined $\mathrm{CD} 3+\mathrm{CD} 4+\mathrm{T}$ and $\mathrm{CD} 3+\mathrm{CD} 8+\mathrm{T}$ cells in aGVHD target organs, and found a significant decrease in the proportion of $\mathrm{CD} 3+\mathrm{CD} 4+\mathrm{T}$ and $\mathrm{CD} 3+\mathrm{CD} 8+\mathrm{T}$ cells in these tissues of mice treated with hAMSCs compared to PBS. MSCs further favor Treg expansion in vitro indirectly by inhibiting dendritic cell maturation, and CD8 $+T$ cell and NK cell expansion [36, 37]. Our observations further demonstrated that the proportion of CD4 + CD25 + Foxp3 + Treg cells was increased after hAMSCs treatment in the liver, spleen, and gut. Our data is consistent with the group that showed that murine $\mathrm{CD} 4^{+} \mathrm{CD} 25^{+} \mathrm{Foxp} 3^{+} \mathrm{Tregs}$ were induced during GVHD after allogeneic BMT, and the induction of these Tregs was positively correlated with the protection of GVHD in mice [38]. GVHD involves a pathophysiology that includes host tissue damage, increased secretion of proinflammatory cytokines (TNF, IFN- - , IL-1, IL-2, and IL-12), and activation of dendritic cells, macrophages, NK cells, and cytotoxic T cells [39]. Inhibition of proinflammatory cytokines has been shown to be beneficial in resolution of the severity and incidence of GVHD $[40,41]$. Although some in vitro studies have suggested that IL-10 or TGF- $\beta$ may be involved in the suppression of MSCs $[42,43]$. it remains unknown whether these molecules participate in the suppression mediated by hAMSCs in vivo. Here, we found that the amounts of IL-17A, IFN-y, TNF, and IL-2 in target organs such as the liver, lung, and gut decreased after hAMSCs treatment. IL-17A was initially reported to be produced by T helper 17 (Th17) cells [44]. In general, pro-inflammatory cytokines such as IFN- $\gamma$, TNF- $a$, IL-1 $a$, or IL-1 $\beta$ have been extensively reported in MSCs activation in vitro [45]. However, there are few studies on inflammatory cytokines by hAMSCs in vivo. Our data also showed that hAMSCs treatment inhibited IL-17A, INF-y, TNF, and IL-2, which are involved in the pathogenesis of GVHD target organs. Th17 cells and Th17-associated cytokines play a central role in the occurrence of aGVHD [46, 47]. IL-17 contributed to the development of aGVHD in recipient mice by recruiting or priming Th1 cells during the early stages of the disease, reflecting a shift from Th1 to Th17 cells in the physiopathology of aGVHD [46]. A subset of Th17 cells in the gut has been described as having regulatory properties with high levels of IL-10 and low TNF and IL-2 production [48]. We found that IL-17A, IFN- $y$ and IL-2 were increased in the gut of the aGVHD model; after hAMSCs treatment, the levels of IL-17A, IFN-yand IL-2 were significantly decreased, which means hAMSCs can suppressed Th1 and Th17 cells in aGVHD mouse model. It has been well-established from both murine studies and immune reconstitution data in the clinic that the production of IFN- $y$ was an adverse effect of GVHD-associated cytokines [49, 50, 51]. Aggarwal et al. suggested that MSCs inhibited IFN- $\gamma$ and increased IL-4 secretion, and may orchestrate a shift from the prominence of proinflammatory Th1 cells toward an increase in anti-inflammatory Th2 cells, beneficial for GVHD management [52]. Other studies have also shown that a high level of IL-2 might favored the exacerbation of T cell-mediated inflammation rather than the survival of Treg cells under proinflammatory conditions [53]. Furthermore, hAMSCs decreased the level of IFN- $y$ in the liver, lung, gut, and blood and decreased the level of IL-2 in the gut and blood simultaneously. The hAMSCs possessed potent immunomodulatory properties capable of suppressing allogeneic $\mathrm{T}$ cell responses in vivo. The immune suppressive activity of hAMSCs in vivo was associated with a significant decrease in Th1 and Th17 cytokines, including IFN-y, TNF, IL-17A, and IL-2. 
In summary, using humanized mice with a complete human immune system, we successfully established a human allogeneic acute GVHD model. Using this model, we demonstrated that hAMSCs could control acute GVHD by regulating the balance of Treg and T effector cells. Our study provided a proof of concept of hAMSCs treatment to control GVHD after BMT. This strategy could be readily extended to human clinical trials using hAMSCs alone or in combination with minimal conventional immunosuppression to control GVHD. Furthermore, our data also demonstrated that the pathogenesis of aGVHD shared important features with human GVHD and that NPG mice could serve as a better model to study GVHD.

\section{Conclusions}

NPG mice are a valuable tool for studying GVHD induced by human immune cells. The hAMSCs could control acute GVHD by decreasing inflammatory cytokine secretion within target organs through modulating the balance of Treg and T effector cells in humanized mice. This strategy could be extended to human clinical trials using human hAMSCs alone or in combination with minimal conventional immunosuppression to control acute GVHD.

\section{Abbreviations}

NPG, NOD-Prkdc ${ }^{\text {scid IL2ry }}{ }^{\text {null; }}$ SCID, severe combined immune deficiency; Prkdc, DNA-dependent protein kinase catalytic subunit; NOD-SCID, non-obese diabetic-SCID; NK, natural killer; IL2ry, interleukin-2 receptor

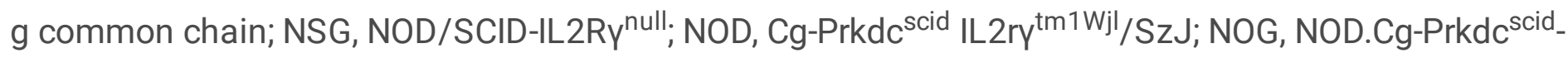

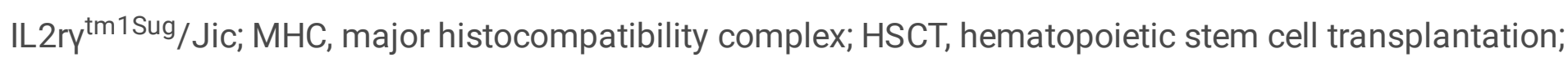
xeno-GVHD, xenogeneic GVHD; GVHD, graft-versus-host disease; MSCs, mesenchymal stem cell; hAMSCs, human amniotic mesenchymal stem cells; BM-MSCs, bone marrow mesenchymal stem cells; PBMCs, peripheral blood mononuclear cells.

\section{Declarations}

\section{Acknowledgements}

The authors thank the teachers in the Department of Laboratory Medicine, Nanfang Hospital, Southern Medical University for technical assistance.

\section{Authors' contributions}

Ping Baohong and Wang Qian conceived and designed the study; and Gao Ya, Li Weiru, Bu Xiaoyin, Cai Shengchun, Zhong Jinman, Xu ying, He Yongjian, and Du Meixue performed the experiments. Huang Liping collected and provided the placentas of healthy women. Gao Ya and Li Weiru wrote the manuscript, Jin Hua, Sun Haitao, and Hu Xiumei reviewed and edited the manuscript. Liu Qifa participated in the discussion. All authors read and approved the final manuscript.

\section{Funding}


The authors gratefully acknowledge the Natural Science Foundation of China $(81701243,81870145$, 81772244), the Science and Technology Planning Project of Guangzhou (201904010481), the Natural Science Fund of Guangdong Province (2020A1515010038), the Presidential Foundation of Nanfang Hospital of Southern Medical University (2017C030), and the Presidential Foundation of Zhujiang Hospital of Southern Medical University (yzjj2018rc03).

\section{Availability of data and materials}

The datasets used and/or analyzed during the current study are available from the corresponding author on reasonable request.

\section{Ethics approval and consent to participate}

The study was approved by the Ethics Committee of Nanfang Hospital, Southern Medical University. Signed informed consent with permission to report individual patient data was obtained from all participants after a detailed description of the purposes of our study.

\section{Consent for publication}

Not applicable.

\section{Competing interests}

The authors declare that they have no competing interests.

\section{Author details}

*These authors contributed equally to this work

${ }^{1}$ Department of Hematology, Nanfang Hospital, Southern Medical University, Guangzhou 510515, China

${ }^{2}$ Department of Hematology, Huiqiao Medical Center, Nanfang Hospital, Southern Medical University, Guangzhou, China

${ }^{3}$ Department of Laboratory Medicine, Nanfang Hospital, Southern Medical University, Guangzhou 510515, China

${ }^{4}$ Department of Laboratory Medicine, Zhujiang Hospital, Southern Medical University, Guangzhou, 510280 Guangdong, China

${ }^{5}$ Department of Obstetrics and Gynecology, Nanfang Hospital, Southern Medical

University, Guangzhou, Guangdong, China

\section{References}


1. Ghimire S, Weber D, Mavin E, Wang XN, Dickinson AM, Holler E. Pathophysiology of GvHD and Other HSCT-Related Major Complications. Front Immunol. 2017;8:79.

2. Blazar BR, Murphy WJ, Abedi M. Advances in graft-versus-host disease biology and therapy. Nat Rev Immunol. 2012;12(6):443-58.

3. Wolf $D$, von Lilienfeld-Toal $M$, Wolf $A M$, Schleuning $M$, von Bergwelt-Baildon $M$, Held $S A$, et al. Novel treatment concepts for graft-versus-host disease. Blood. 2012;119(1):16-25.

4. Tan Y, Xiao H, Wu D, Luo Y, Lan J, Liu Q, et al. Combining therapeutic antibodies using basiliximab and etanercept for severe steroid-refractory acute graft-versus-host disease: A multi-center prospective study. Oncoimmunology. 2017;6(3):e1277307.

5. Insausti CL, Blanquer M, García-Hernández AM, Castellanos G, Moraleda JM. Amniotic membranederived stem cells: immunomodulatory properties and potential clinical application. Stem Cells Cloning. 2014;7:53-63.

6. Ono M, Ohnishi S, Honda M, Ishikawa M, Hosono H, Onishi R, et al. Effects of human amnion-derived mesenchymal stromal cell transplantation in rats with radiation proctitis. Cytotherapy. 2015;17(11):1545-59.

7. Hong JQ, Gao Y, Song J, Zhuo WB, Sun HT, Ping BH. Comparison of Biological Characteristics and Immunosuppressive Activity between Human Amniotic Mesenchymal Stem Cells and Human Bone Marrow Mesenchymal Stem Cells. Zhongguo Shi Yan Xue Ye Xue Za Zhi. 2016;24(3):858-64.

8. Yamahara K, Hamada A, Soma T, Okamoto R, Okada M, Yoshihara S, et al. Safety and efficacy of amnion-derived mesenchymal stem cells (AM01) in patients with steroid-refractory acute graftversus-host disease after allogeneic haematopoietic stem cell transplantation: a study protocol for a phase I/II Japanese trial. BMJ Open. 2019;9(7):e026403.

9. Song J, Gao Y, Zhou WB, Yang CY, Xu Y, Ping BH, et al. Immunomodulatory effects of human amniotic versus bone marrow-derived mesenchymal stem cells on peripheral blood T lymphocytes in vitro. Nan Fang Yi Ke Da Xue Xue Bao. 2017;37(6):780-5.

10. Schroeder MA, DiPersio JF. Mouse models of graft-versus-host disease: advances and limitations. Dis Model Mech. 2011;4(3):318-33.

11. Lucas PJ, Shearer GM, Neudorf S, Gress RE. The human antimurine xenogeneic cytotoxic response. I. Dependence on responder antigen-presenting cells. J Immunol. 1990;144(12):4548-54.

12. Ito R, Takahashi T, Katano I, Ito M. Current advances in humanized mouse models. Cell Mol Immunol. 2012;9(3):208-14.

13. Godoy JAP, Paiva RMA, Souza AM, Kondo AT, Kutner JM, Okamoto OK. Clinical translation of mesenchymal stromal cell therapy for graft versus host disease. Front Cell Dev Biol. 2019;7:255.

14. Lim JE, Son Y. Endogenous stem cells in homeostasis and aging. Tissue Eng Regen Med. 2017;14(6):679-98.

15. Knuth CA, Kiernan $\mathrm{CH}$, Palomares Cabeza V, Lehmann J, Witte-Bouma J, Ten Berge D, et al. Isolating pediatric mesenchymal stem cells with enhanced expansion and differentiation capabilities. Tissue Eng Part C Methods. 2018;24(6):313-21. 
16. Umezawa A, Hasegawa A, Inoue M, Tanuma-Takahashi A, Kajiwara K, Makino H, et al. Amnionderived cells as a reliable resource for next-generation regenerative medicine. Placenta. 2019;84:506.

17. King MA, Covassin L, Brehm MA, Racki W, Pearson T, Leif J, et al. Human peripheral blood leucocyte non-obese diabetic-severe combined immunodeficiency interleukin-2 receptor gamma chain gene mouse model of xenogeneic graft-versus-host-like disease and the role of host major histocompatibility complex. Clin Exp Immunol. 2009;157(1):104-18.

18. King M, Pearson T, Shultz LD, Leif J, Bottino R, Trucco M, et al. A new Hu-PBL model for the study of human islet alloreactivity based on NOD-scid mice bearing a targeted mutation in the IL-2 receptor gamma chain gene. Clin Immunol. 2008;126(3):303-14.

19. Zeiser R, Blazar BR. Acute graft-versus-host disease - Biologic process, prevention, and therapy. $\mathrm{N}$ Engl J Med. 2017;377(22):2167-79.

20. Yang X, Zhou J, He J, Liu J, Wang H, Liu Y, et al. An immune system-modified rat model for human stem cell transplantation research. Stem Cell Reports. 2018;11(2):514-21.

21. Hill GR, Crawford JM, Cooke KR, Brinson YS, Pan L, Ferrara JL. Total body irradiation and acute graftversus-host disease: the role of gastrointestinal damage and inflammatory cytokines. Blood. 1997;90(8):3204-13.

22. Schwarte S, Hoffmann MW. Influence of radiation protocols on graft-vs-host disease incidence after bone-marrow transplantation in experimental models. Methods Mol Med. 2005;109:445-58.

23. Mareschi K, Castiglia S, Sanavio F, Rustichelli D, Muraro M, Defedele D, et al. Immunoregulatory effects on T lymphocytes by human mesenchymal stromal cells isolated from bone marrow, amniotic fluid, and placenta. Exp Hematol. 2016;44(2):138-50.e1.

24. Sun H, Hou Z, Yang H, Meng M, Li P, Zou Q, et al. Multiple systemic transplantations of human amniotic mesenchymal stem cells exert therapeutic effects in an ALS mouse model. Cell Tissue Res. 2014;357(3):571-82.

25. Di Nicola M, Carlo-Stella C, Magni M, Milanesi M, Longoni PD, Matteucci P, et al. Human bone marrow stromal cells suppress T-lymphocyte proliferation induced by cellular or nonspecific mitogenic stimuli. Blood. 2002;99(10):3838-43.

26. Blanco B, Herrero-Sánchez MD, Rodríguez-Serrano C, García-Martínez ML, Blanco JF, Muntión S, et al. Immunomodulatory effects of bone marrow versus adipose tissue-derived mesenchymal stromal cells on NK cells: implications in the transplantation setting. Eur J Haematol. 2016;97(6):528-37.

27. Huang Y, Yin Y, Gu Y, Gu Q, Yang H, Zhou Z, et al. Characterization and immunogenicity of bone marrow-derived mesenchymal stem cells under osteoporotic conditions. Sci China Life Sci. 2020;63(3):429-42.

28. Keith MC, Bolli R. "String theory" of c-kit(pos) cardiac cells: a new paradigm regarding the nature of these cells that may reconcile apparently discrepant results. Circ Res. 2015;116(7):1216-30.

29. Eseonu OI, De Bari C. Homing of mesenchymal stem cells: mechanistic or stochastic? Implications for targeted delivery in arthritis. Rheumatology. 2015;54(2):210-8. 
30. Nitzsche F, Müller C, Lukomska B, Jolkkonen J, Deten A, Boltze J. Concise Review: MSC adhesion cascade-insights into homing and transendothelial migration. Stem Cells. 2017;35(6):1446-60.

31. Thompson M, Mei SHJ, Wolfe D, Champagne J, Fergusson D, Stewart DJ, et al. Cell therapy with intravascular administration of mesenchymal stromal cells continues to appear safe: An updated systematic review and meta-analysis. E Clinical Medicine. 2020;19:100249.

32. Thangavelu G, Blazar BR. Achievement of tolerance induction to prevent acute graft-vs.-host disease. Front Immunol. 2019;10:309.

33. Tobin LM, Healy ME, English K, Mahon BP. Human mesenchymal stem cells suppress donor CD4(+) $T$ cell proliferation and reduce pathology in a humanized mouse model of acute graft-versus-host disease. Clin Exp Immunol. 2013;172(2):333-48.

34. Amorin B, Alegretti AP, Valim V, Pezzi A, Laureano AM, da Silva MA, et al. Mesenchymal stem cell therapy and acute graft-versus-host disease: a review. Hum Cell. 2014;27(4):137-50.

35. Tago Y, Kobayashi C, Ogura M, Wada J, Yamaguchi S, Yamaguchi T, et al. Human amnion-derived mesenchymal stem cells attenuate xenogeneic graft-versus-host disease by preventing $T$ cell activation and proliferation. Sci Rep. 2021;11(1):2406.

36. Cahill EF, Tobin LM, Carty F, Mahon BP, English K. Jagged-1 is required for the expansion of CD4 + CD25 + FoxP3 + regulatory T cells and tolerogenic dendritic cells by murine mesenchymal stromal cells. Stem Cell Res Ther. 2015;6(1):19.

37. Melief SM, Schrama E, Brugman MH, Tiemessen MM, Hoogduijn MJ, Fibbe WE, et al. Multipotent stromal cells induce human regulatory $T$ cells through a novel pathway involving skewing of monocytes toward anti-inflammatory macrophages. Stem Cells. 2013;31(9):1980-91.

38. Koreth J, Ritz J, Tregs. HSCT, and acute GVHD: up close and personal. Blood. 2013;122(10):1690-1.

39. Dong W, Wu X, Ma S, Wang Y, Nalin AP, Zhu Z, et al. The mechanism of anti-PD-L1 antibody efficacy against PD-L1-negative tumors identifies NK cells expressing PD-L1 as a cytolytic effector. Cancer Discov. 2019;9(10):1422-37.

40. Bruner RJ, Farag SS. Monoclonal antibodies for the prevention and treatment of graft-versus-host disease. Semin Oncol. 2003;30(4):509-19.

41. Choi SW, Reddy P. Current and emerging strategies for the prevention of graft-versus-host disease. Nat Rev Clin Oncol. 2014;11(9):536-47.

42. Ng J, Hynes K, White G, Sivanathan KN, Vandyke K, Bartold PM, et al. Immunomodulatory properties of induced pluripotent stem cell-derived mesenchymal cells. J Cell Biochem. 2016;117(12):2844-53.

43. El-Sherbiny YM, El-Jawhari JJ, Moseley TA, McGonagle D, Jones E. T cell immunomodulation by clinically used allogeneic human cancellous bone fragments: a potential novel immunotherapy tool. Sci Rep. 2018;8(1):13535.

44. Steinman L. A brief history of $T(H) 17$, the first major revision in the $T(H) 1 / T(H) 2$ hypothesis of $T$ cellmediated tissue damage. Nat Med. 2007;13(2):139-45. 
45. Cheung TS, Bertolino GM, Giacomini C, Bornhäuser M, Dazzi F, Galleu A. Mesenchymal Stromal Cells for Graft Versus Host Disease: Mechanism-Based Biomarkers. Front Immunol. 2020;11:1338.

46. Kim KW, Moon SJ, Park MJ, Kim BM, Kim EK, Lee SH, et al. Optimization of adipose tissue-derived mesenchymal stem cells by rapamycin in a murine model of acute graft-versus-host disease. Stem Cell Res Ther. 2015;6:202.

47. Gartlan KH, Markey KA, Varelias A, Bunting MD, Koyama M, Kuns RD, et al. Tc17 cells are a proinflammatory, plastic lineage of pathogenic CD8 + T cells that induce GVHD without antileukemic effects. Blood. 2015;126(13):1609-20.

48. Esplugues E, Huber S, Gagliani N, Hauser AE, Town T, Wan YY, et al. Control of TH17 cells occurs in the small intestine. Nature. 2011;475(7357):514-8.

49. Capitini CM, Herby S, Milliron M, Anver MR, Mackall CL, Fry TJ. Bone marrow deficient in IFN\{gamma\} signaling selectively reverses GVHD-associated immunosuppression and enhances a tumor-specific GVT effect. Blood. 2009;113(20):5002-9.

50. Merli P, Caruana I, De Vito R, Strocchio L, Weber G, Bufalo FD, et al. Role of interferon-y in immunemediated graft failure after allogeneic hematopoietic stem cell transplantation. Haematologica. 2019;104(11):2314-23.

51. Alvarado LJ, Huntsman HD, Cheng H, Townsley DM, Winkler T, Feng X, et al. Eltrombopag maintains human hematopoietic stem and progenitor cells under inflammatory conditions mediated by IFN- $\gamma$. Blood. 2019;133(19):2043-55.

52. Aggarwal S, Pittenger MF. Human mesenchymal stem cells modulate allogeneic immune cell responses. Blood. 2005;105(4):1815-22.

53. Ratnasothy K, Jacob J, Tung S, Boardman D, Lechler RI, Sanchez-Fueyo A, et al. IL-2 therapy preferentially expands adoptively transferred donor-specific Tregs improving skin allograft survival. Am J Transplant. 2019;19(7):2092-100.

\section{Tables}

Due to technical limitations, table 1 is only available as a download in the Supplemental Files section.

\section{Figures}


A

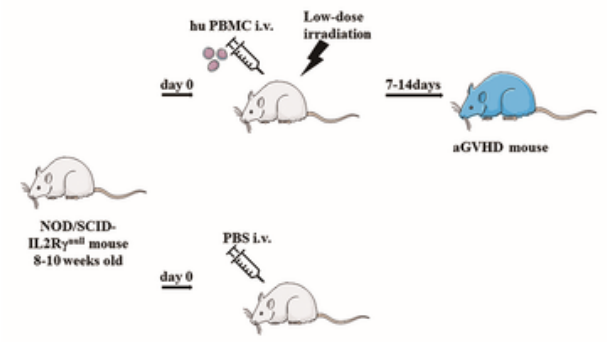

B
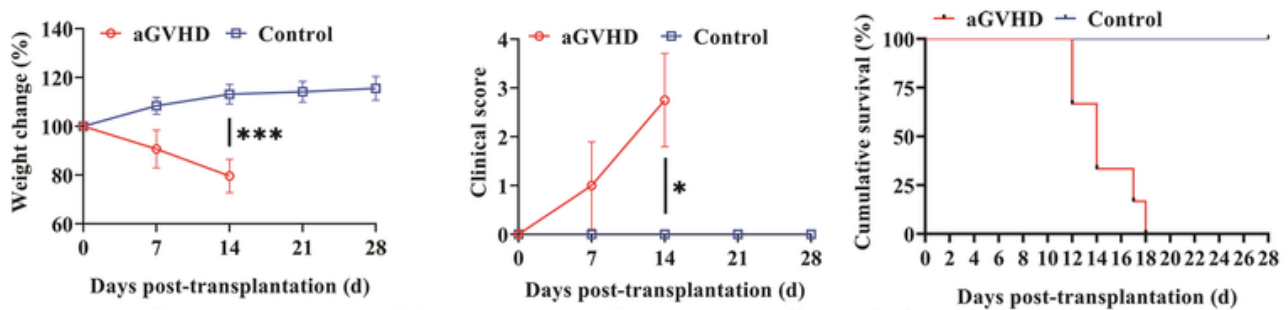

C
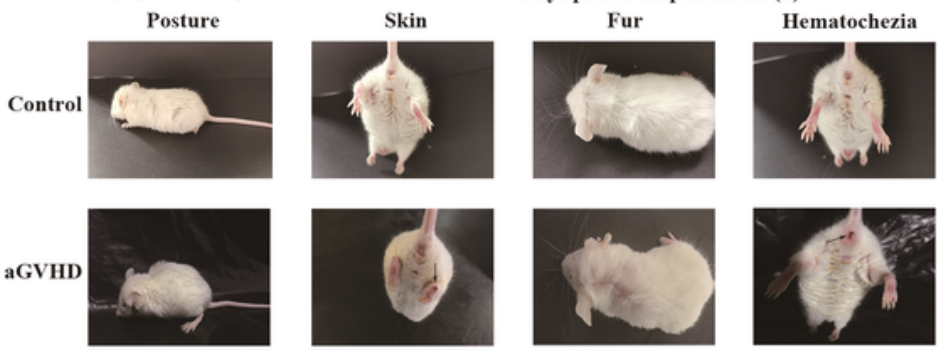

D
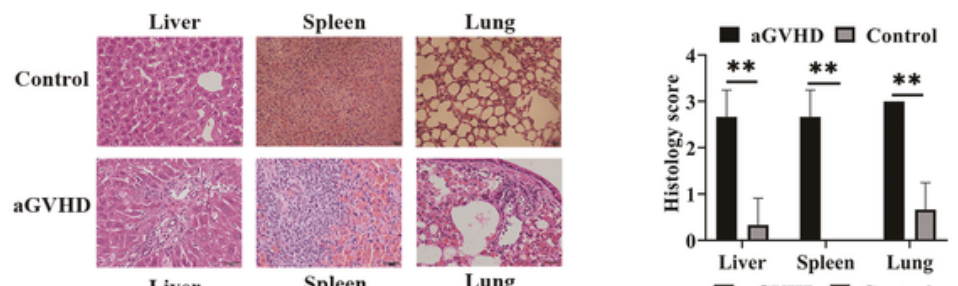

E
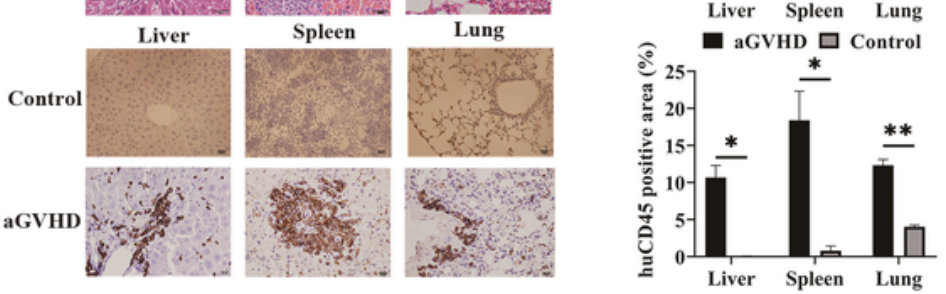

F

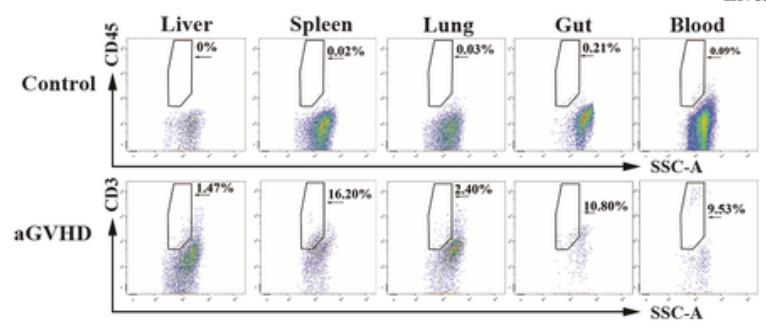

\section{Figure 1}

Low dose irradiation before PBMCs injection induced xeno-aGVHD in NPG mice. (A) Establishment of the xeno-aGVHD model. NPG mice were injected with allogeneic hPBMCs (aGVHD group) or PBS (control group) following sublethal irradiation. (B) Measurement of weight change, clinical score, and survival time at different time points with those two groups. The weight loss rate in aGVHD group is faster than in the control group, the clinical score of aGVHD was higher than that of the control group, The survival time 
of the control group was also significantly longer than that of the aGVHD group. (C) Representative clinical manifestations including hunched posture, ruffed fur, damaged skin, and hematochezia in the xeno-aGVHD mouse model. (D) Representative histology of target organs, including the liver, spleen, and lung, showed inflammatory cell infiltration and tissue damage in the low-dose irradiation pre-conditioned NPG mice group. $400 \times .{ }^{*} p<0.05,{ }^{*} p<0.01$, and ${ }^{* * *} p<0.001$. (E) Representative immunohistochemistry of target organs. Inflammatory cell infiltration and tissue damage in target organs such as the lung, liver, and spleen. Human CD45+ cells were also detected by immunohistochemistry in these target organs. 400x. ${ }^{*} p<0.05,{ }^{*} p<0.01$, and ${ }^{* \star *} p<0.001$. (F) Human CD 45+ T cells were not detected by flow cytometry in the control group. Human $\mathrm{CD} 3+\mathrm{T}$ cells from each target organ were detected by flow cytometry in the aGVHD group.

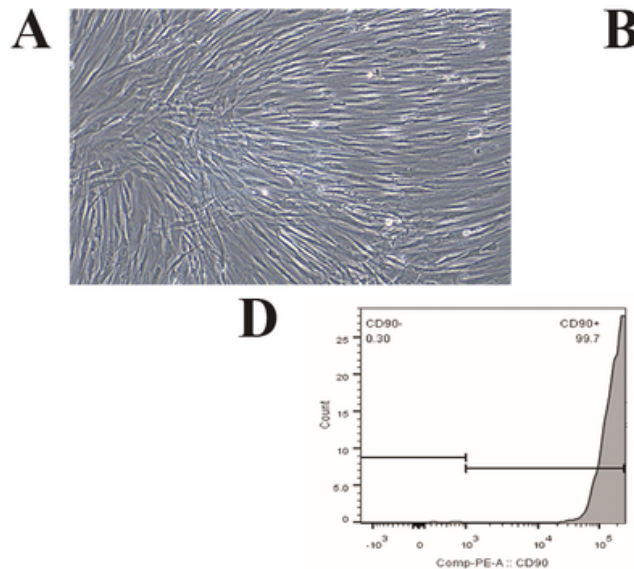

CD90+
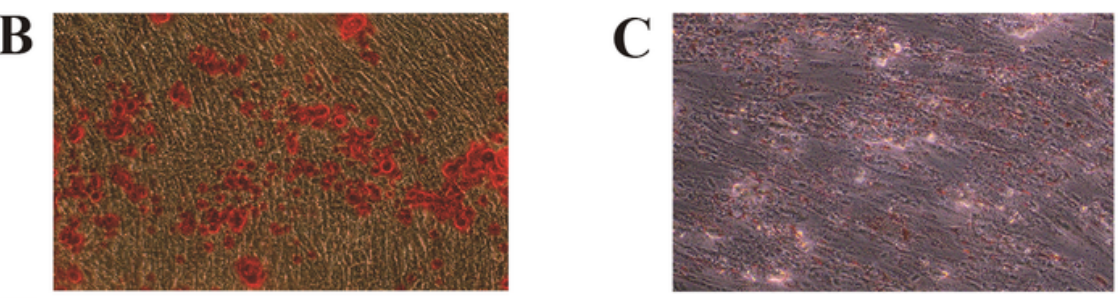

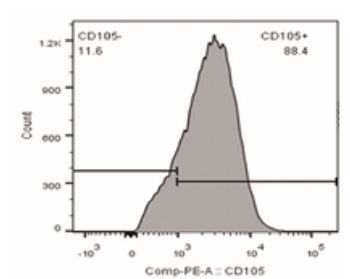

CD105+

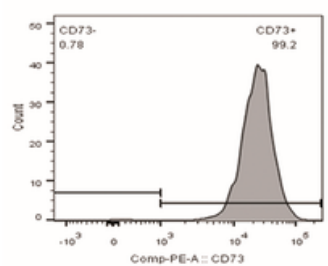

CD73+

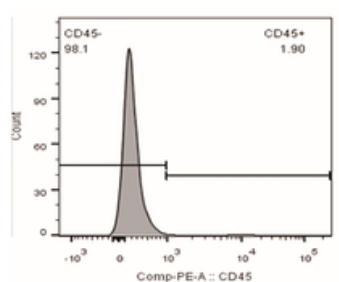

CD45-

E

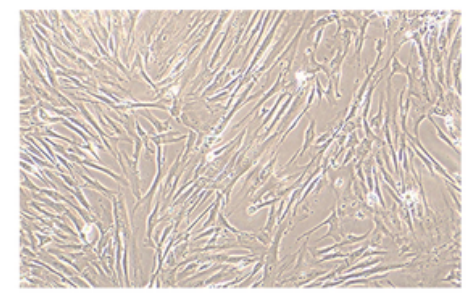

Control

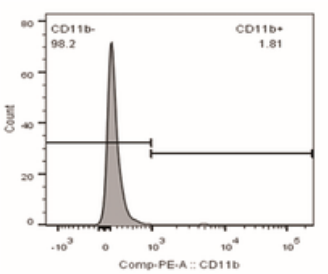

CD11b-

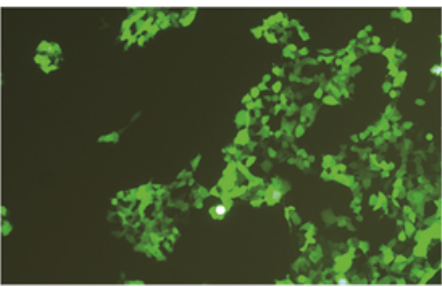

GFP-293T

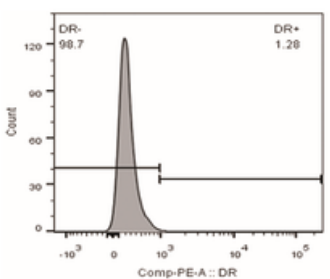

HLA-DR-

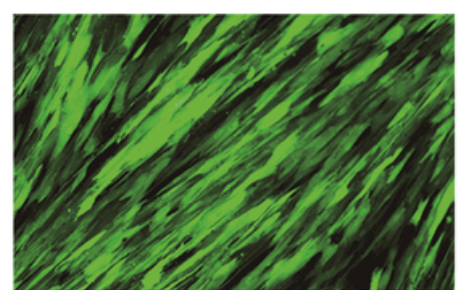

GFP-hAMSCs

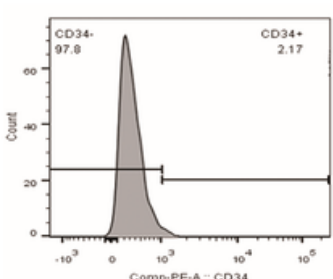

CD34-

\section{Figure 2}

Identification of human amnion mesenchymal stem cells. (A) Characteristics of passage three hAMSCs. (B) Osteogenic differentiation of hAMSCs indicated by Alizarin Red S. 100x. (C) Adipogenic differentiation of hAMSCs indicated by Oil Red O staining. 100x. (D) Surface antigens of hAMSCs were detected by flow cytometry. Cells were positive for CD90, CD105, and CD73 and negative for CD45, CD11b, CD34, and HLA-DR. (E) GFP-labeled hAMSCs and 293T cells were detected by fluorescence 
microscopy at day 3 following transfection with GFP-pseudovirion. $100 \times$. ${ }^{\star} p<0.05$, ${ }^{\star \star} p<0.01$, and ${ }^{\star \star *} p<$ 0.001 .

24h
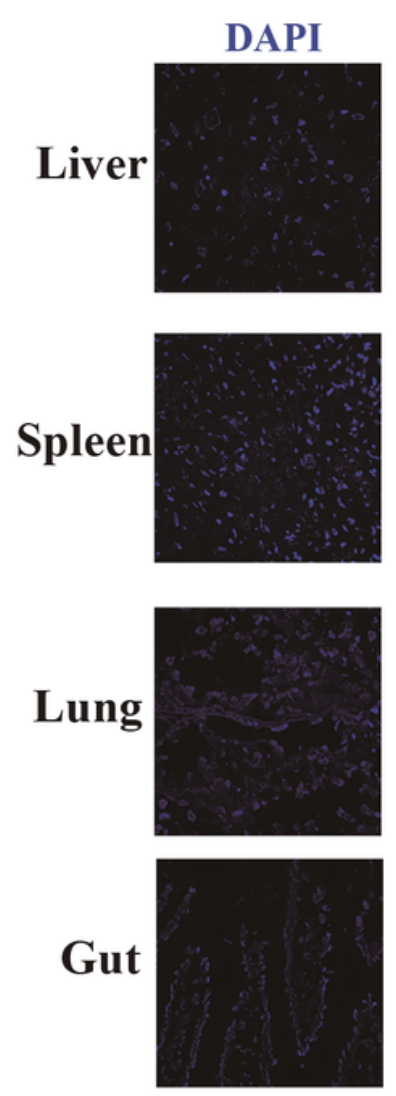
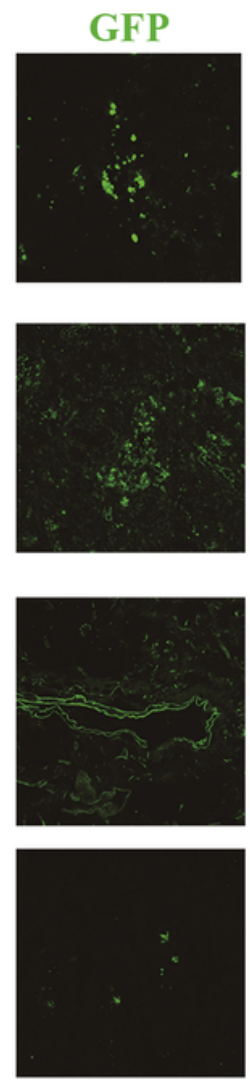

Merge
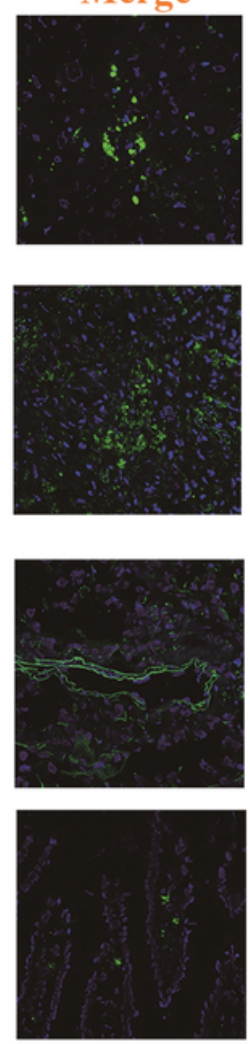

$72 \mathrm{~h}$

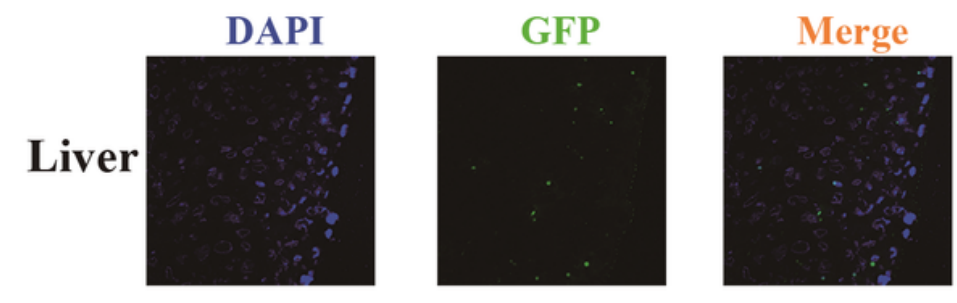

Spleen
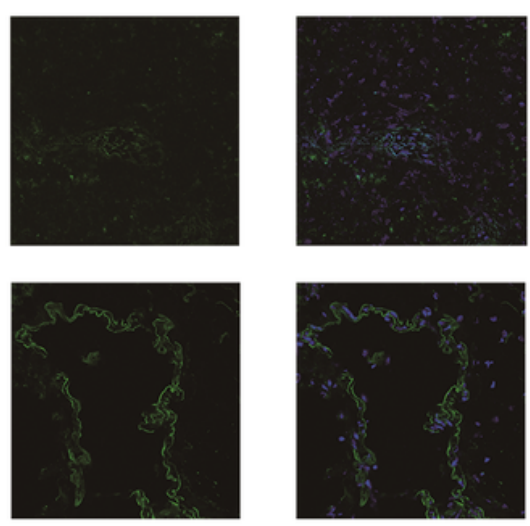

Gut
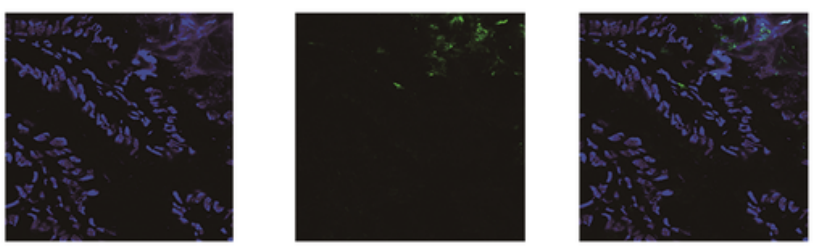

Figure 3

Human amnion mesenchymal stem cells migrated to target organs. GFP-labeled hAMSCs detected by confocal microscopy in target organs at 24 after injection in vivo. At $72 \mathrm{~h}$, GFP fluorescence in the spleen, liver, and gut was significantly weakened while increased in the lung. 
A
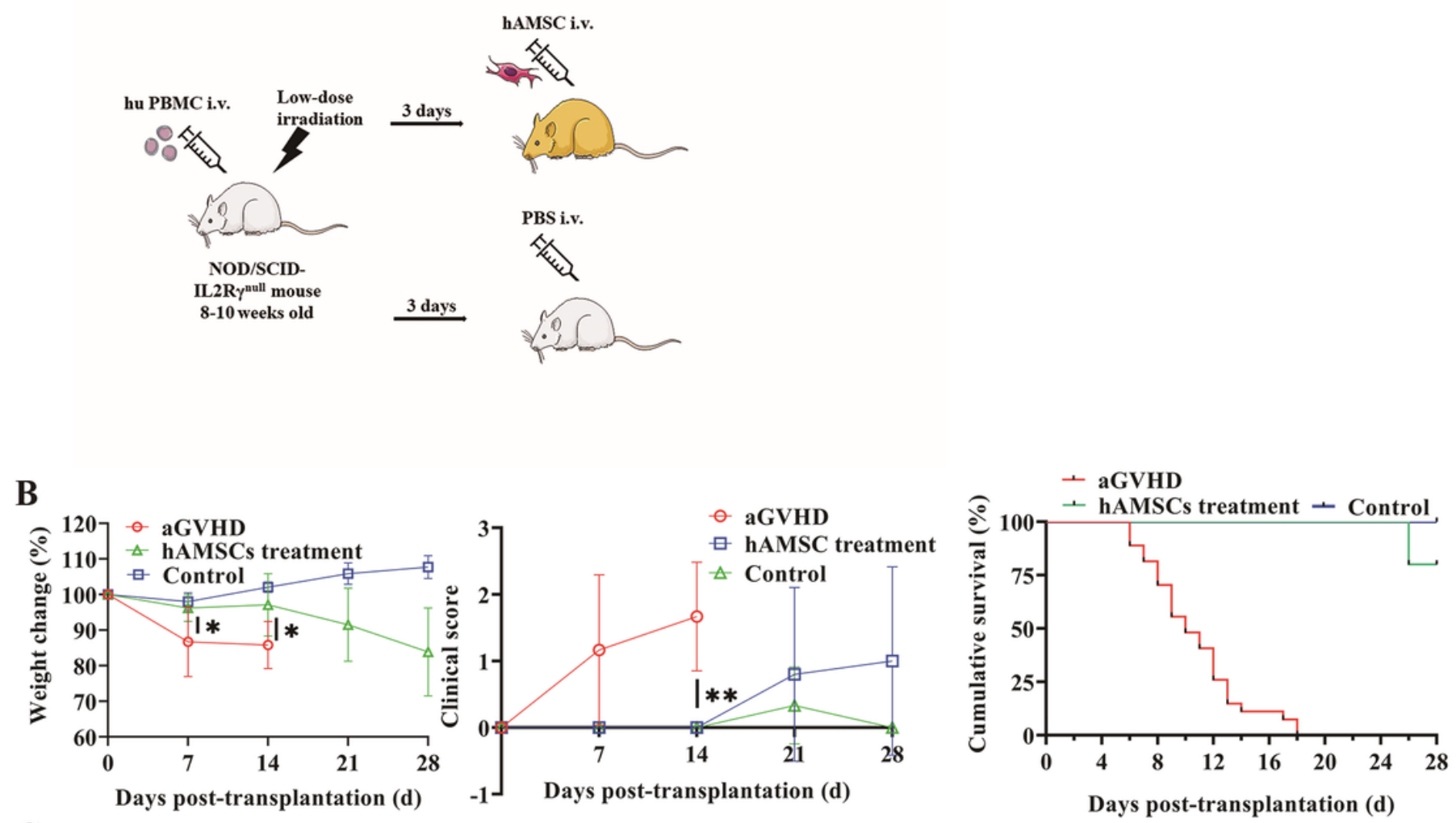

C

Liver

Spleen
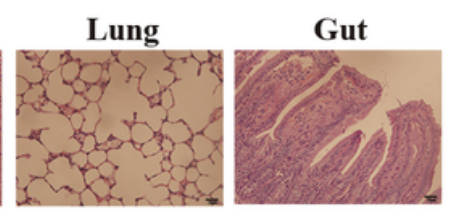

aGVHD
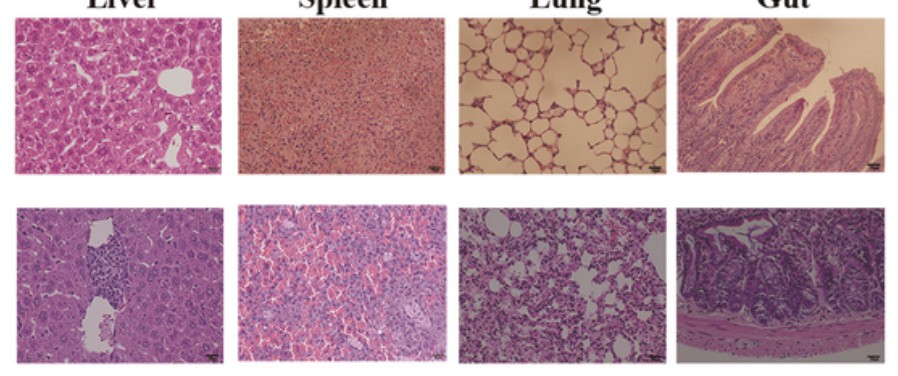

hAMSCs treatment
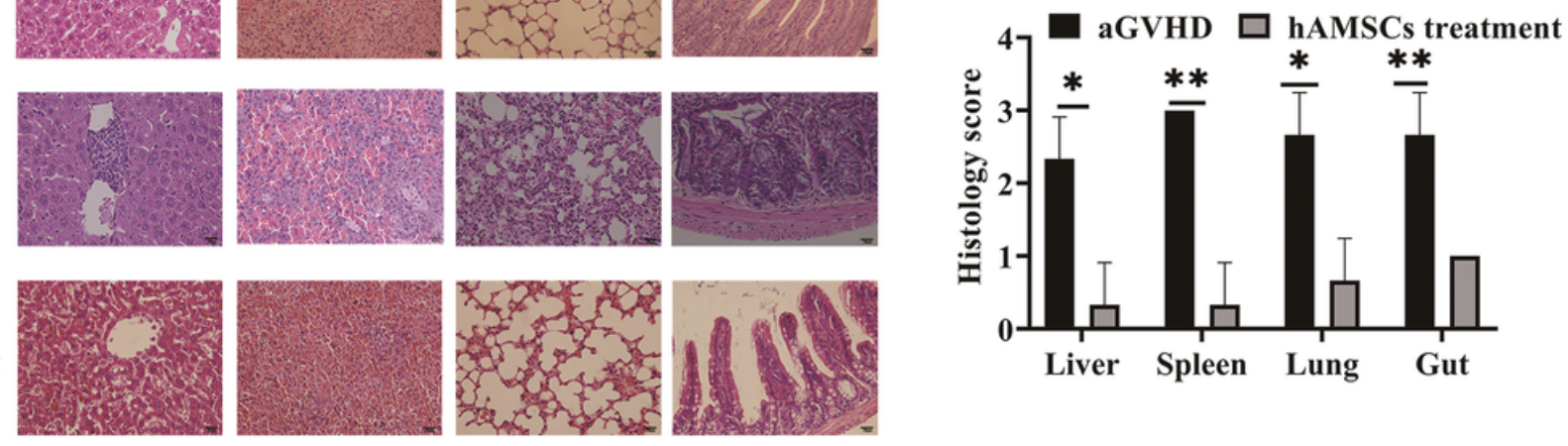

Figure 4

The hAMSCs significantly inhibited the development of xeno-aGVHD. (A) Protocol to determine the immunosuppressed ability of hAMSCs in vivo. Mice were divided into control group (untreated), aGVHD group (injection of PBS three days after modeling), and hAMSCs treatment group (injection of hAMSCs three days after modeling). (B) Measurement of weight change, clinical score, and survival in the different groups. The hAMSCs could significantly slow down the development of aGVHD. (C) Representative histology of target organs, including the liver, spleen, lung, and gut. Compared with the hAMSCs treatment group, inflammatory cell infiltration and tissue damage in the aGVHD group were more severe. 400x. ${ }^{*}<$ $0.05, * * p<0.01$, and $* * * p<0.001$. 
A

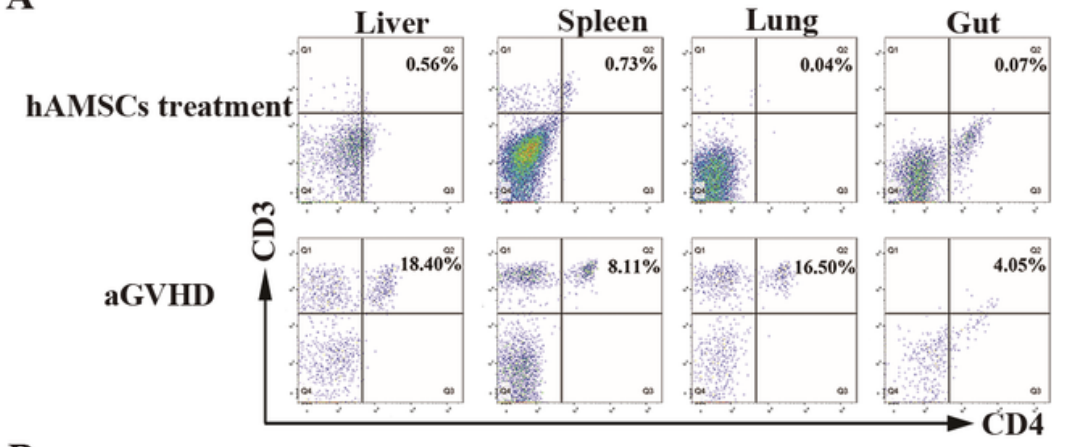

B

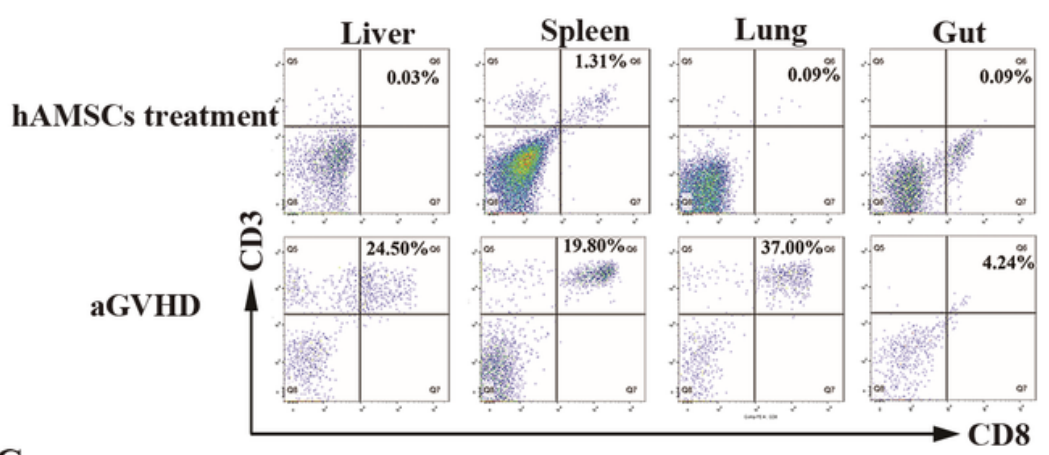

C

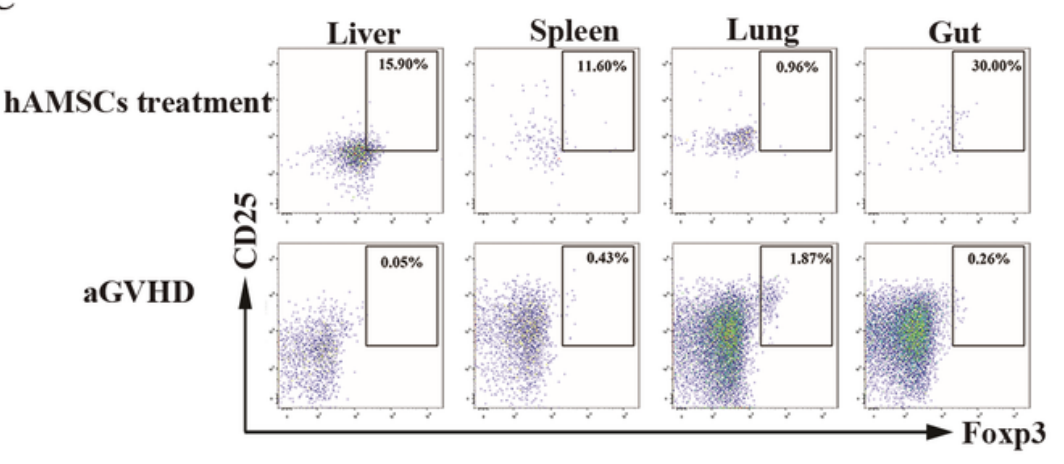

$\square$ aGVHD $\square$ hAMSCs treatment
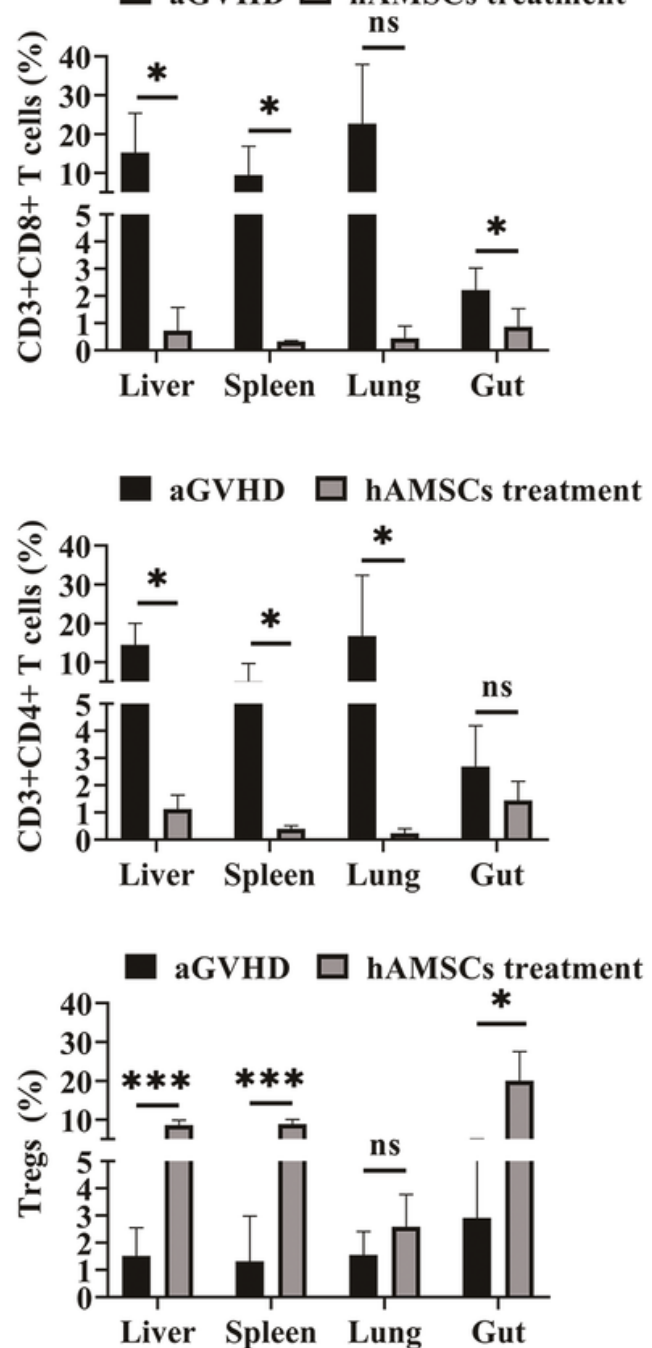

Figure 5

The hAMSCs suppressed CD3+CD4+ T and CD3+CD8+ T cells and increased the proportion of CD4+CD25+Foxp3+ Treg cells. (A) After hAMSCs treatment, the proportion of CD3+CD4+ T cells in liver, spleen, and lung was significantly lower than that of aGVHD group. ${ }^{*} p<0.05$, ${ }^{*} p<0.01$, and ${ }^{* \star *} p<$ 0.001. (B) After hAMSCs treatment, the proportion of CD3+CD8+ $T$ in liver, spleen and gut was significantly lower than that of aGVHD group. ${ }^{*} p<0.05, * \star p<<0.01$, and $* \star * p<0.001$. (C) After hAMSCs treatment,the proportion of CD4+CD25+Foxp3+ Treg cells in liver, spleen, and gut was significantly increased compared with that of aGVHD group. ${ }^{*} p<0.05$, ${ }^{\star *} p<0.01$, and ${ }^{* \star *} \mathrm{p}<0.001$. 
A

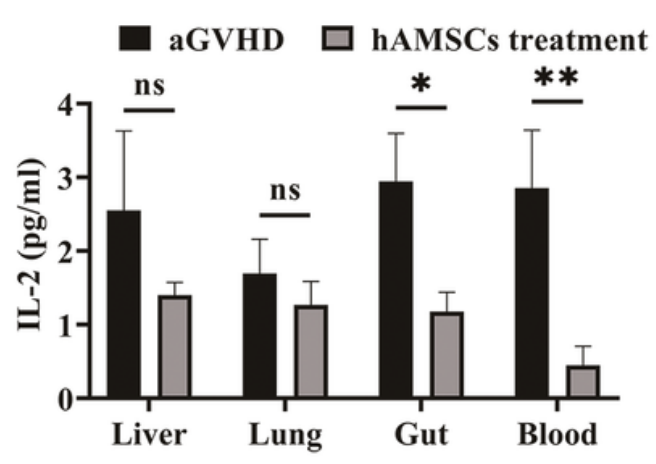

C

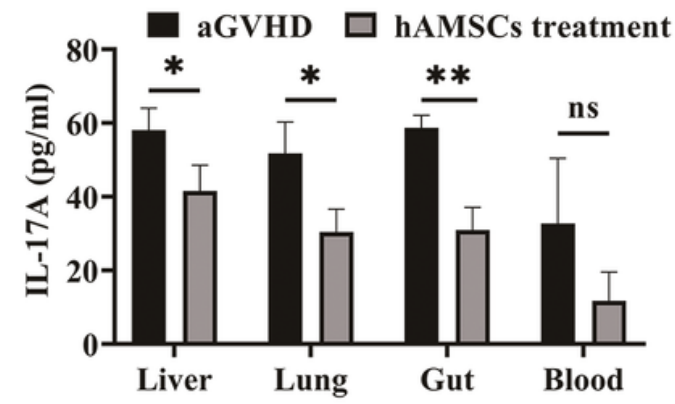

B aGVHD $\square$ hAMSCs treatment

D
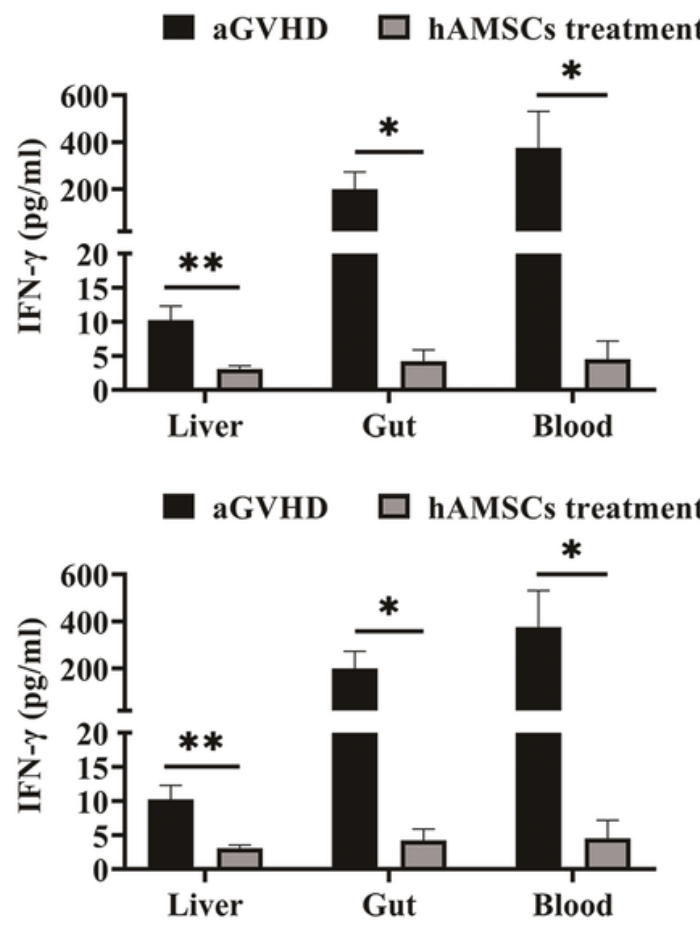

Figure 6

The hAMSCs inhibited cytosine secretion in target organs after hPBMCs transplantation. (A) IL-2 level in gut and blood were significantly decreased in hAMSCs treatment group than that in aGVHD group. ${ }^{*} \mathrm{p}<$ $0.05,{ }^{* *} p<0.01$, and ${ }^{* *} \mathrm{p}<0.001$. (B) IFN-y level in liver, gut and blood were significantly decreased in hAMSCs treatment group than that in aGVHD group. ${ }^{*} p<0.05,{ }^{*} p<0.01$, and ${ }^{* * *} p<0.001$. (C) IL-17A level in liver, lung and gut were significantly decreased in hAMSCs treatment group than that in aGVHD group. ${ }^{*} p<0.05,{ }^{* *} p<0.01$, and ${ }^{* *} p<0.001$. (D) TNF level in liver and lung were significantly decreased in hAMSCs treatment group than that in aGVHD group. Data are means \pm SEM of three independent experiments. ${ }^{*} p<0.05,{ }^{*} p<0.01$, and $* * * p<0.001$.

\section{Supplementary Files}

This is a list of supplementary files associated with this preprint. Click to download.

- Table1.xIsx 